O. Baur'1, H. Bock², P. Ditmar³, H. Hashemi Farahani³, A. Jäggi², T. Mayer-Gürr4, T. Reubelt ${ }^{5}$, N. Zehentner ${ }^{4}$

(1) Space Research Institute, Austrian Academy of Sciences, Graz (2) Astronomical Institute, University of Bern, Switzerland (3) Delft Institute for Earth-Oriented Space research, Delft University
of Technology, The Netherlands (4) Institute of Theoretical Geodesy and Satellite Geodesy, Graz University of Technology, Austria (5) Institute of Geodesy, University of Stuttgart, Germany

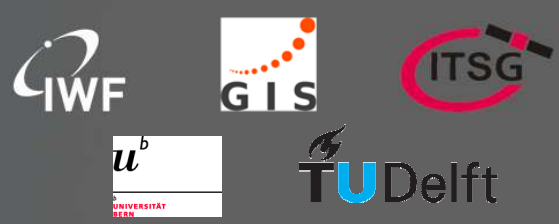

\title{
Summary
}

Several approaches have been proposed to extract gravity field information from the GPS-derived kinematic GOCE (Gravity field and steady-state Ocean Circulation Explorer) orbits. Although there is a general consensus that, except for energy balance, these methods theoretically provide equivalent results, GOCE-GPS solutions based on real data have never been compared with each other within a consistent data processing environment so far. This contribution strives to close this gap. The gravity field solutions considered here make use of the

CMA Celestial Mechanics Approach [1] computed at AIUB (U Bern)

SAA Short-Arc Approach [2] computed at ITSG (TU Graz)

AAA Averaged Acceleration Approach [3] computed at DEOS (TU Delft)

PAA Point-wise Acceleration Approach [4] computed at GIS/IWF (U Stuttgart/Austrian Acad. of Sciences)

EBA Energy Balance Approach [5]

computed at INAS (TU Graz)

Formal errors; empirical errors \& geoid height differences (w.r.t. ITG-Grace2010s)

CMA

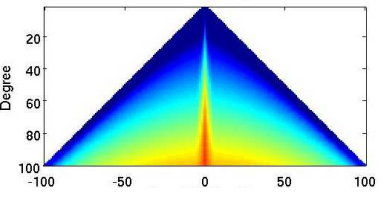

SAA

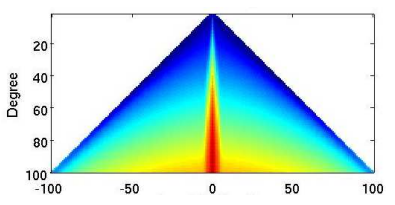

AAA

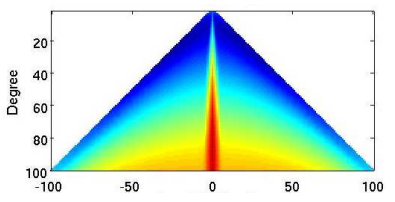

PAA

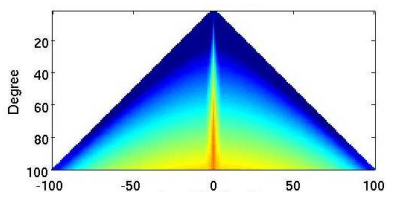

EBA

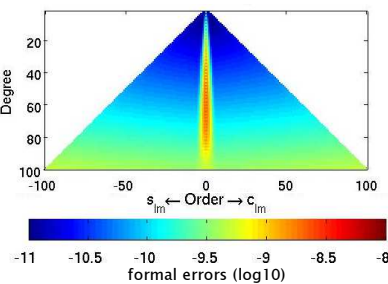

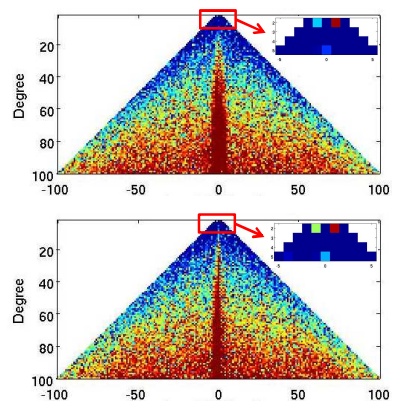
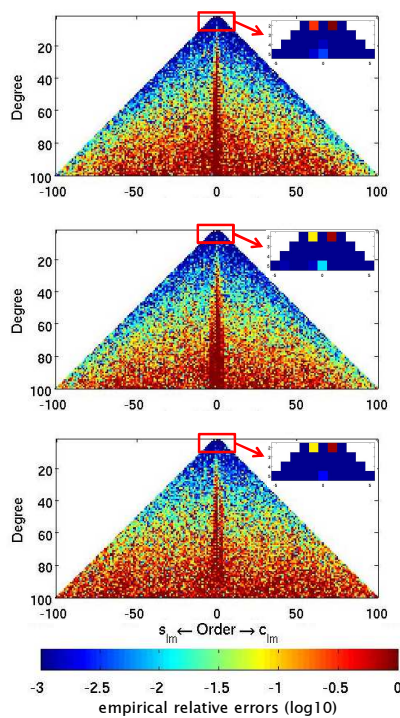
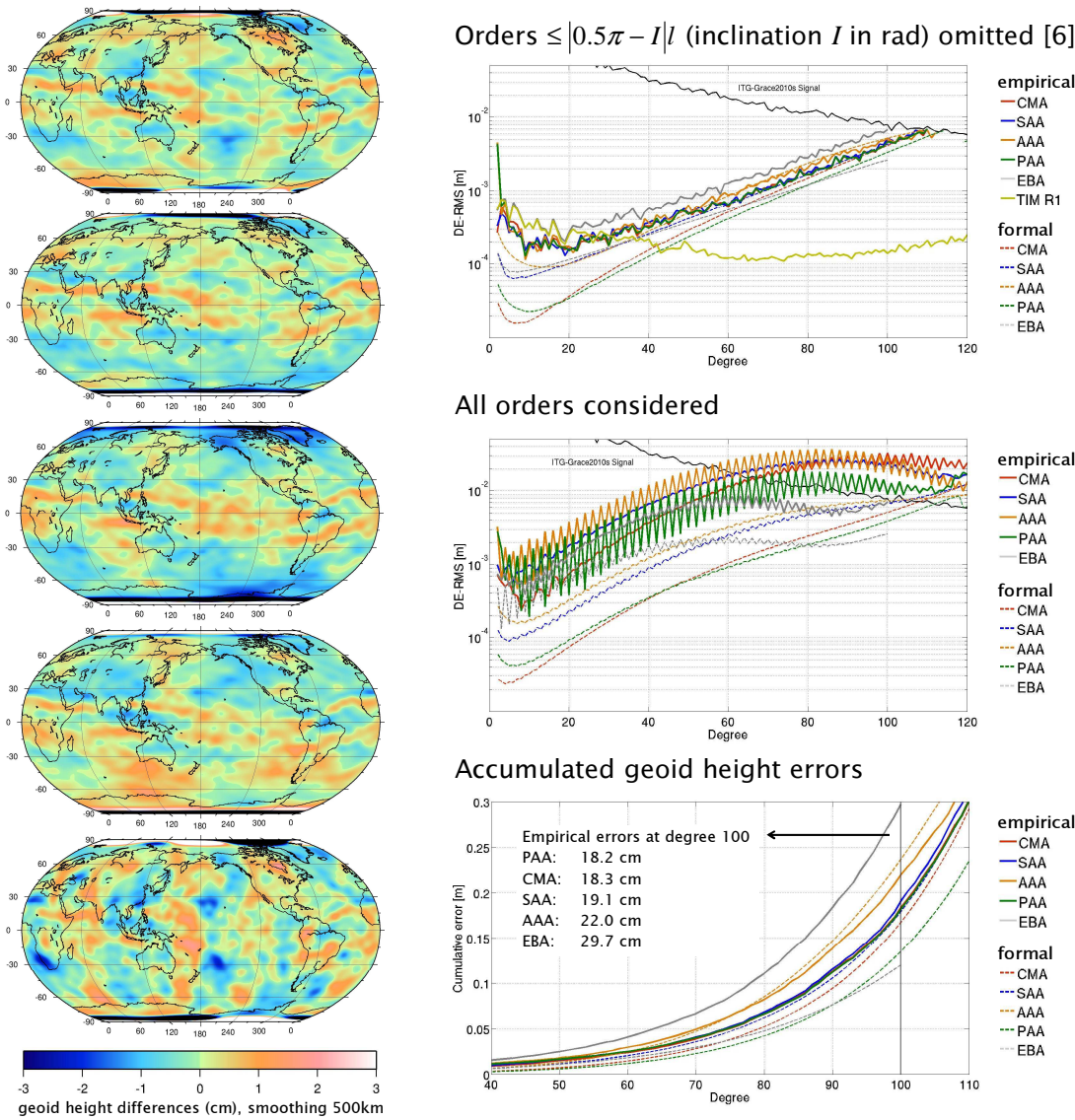

All orders considered

Non-gravitational accel.

Empirical accelerations yes

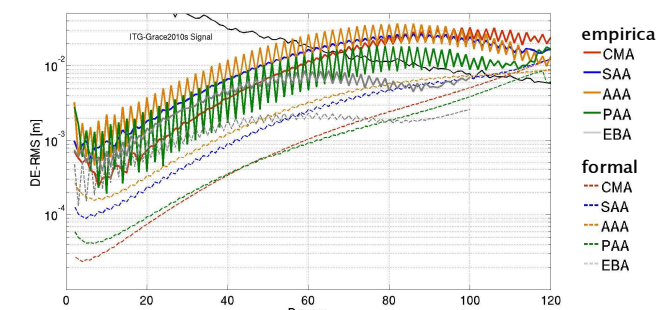

Accumulated geoid height errors

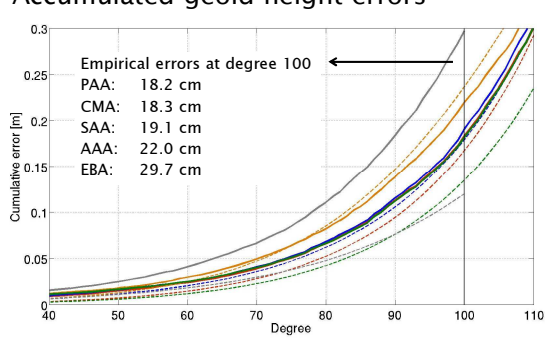

Processing details

Orbit data

Variance-covariance data

Period

Spectral resolution

Regularization

A priori information

Background models

CMA

SAA

AAA

PAA

EBA

ESA SST PKI Prod ESA SST_PCV product

kinematic GOCE orbit)

ESA SST_PCV product

$1.11 .2009-11.1 .2010(\mathrm{R} 1)$

120

100

EGM96

according to IERS Conventions 2003/2010

no yes

\section{SLR tracking residuals (obs.-comp.)}

Parameterization: monthly arcs

Estimated parameters: state vectors ( $1 /$ arc), station coordinates accelerations ( $1 /$ day), measurement biases ( $1 /$ station and arc)

Lageos 1 (up to degree and order 20)

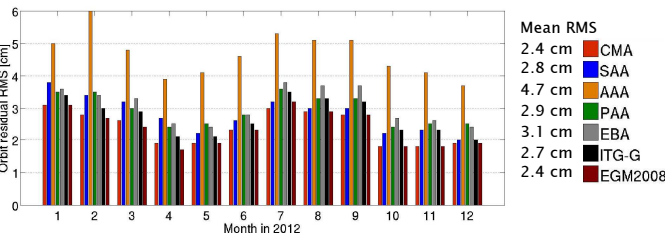

c20 coefficient replaced by SLR-derived value

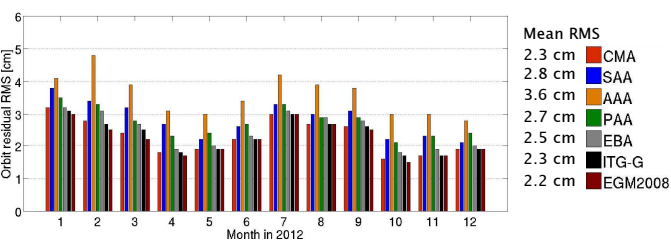

Acknowledgements \& References

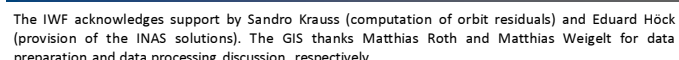

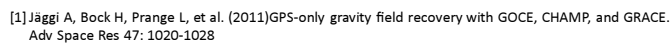

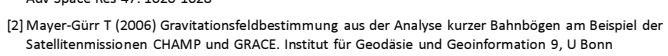
[3] Ditmar P, van Eck van der Sliijs A (2004) A technique for modeling the Earth's gravity field on the
basis of satellite accelerations. $J$ Geod $78: 12-33$

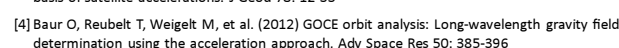
[5] Pail R, Bruinsma S, Migliaccio F, et al (2011) First GocE gravity field models derived by three different
approaches. J Geod 85: 819-843

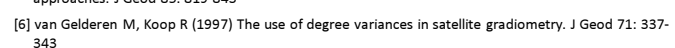
EGU General Assembly 2013, Vienna, Austria, 07-12 April 2013 